Revista Colombiana de Obstetricia y Ginecología Vol. 64 No. 4 • Octubre-Diciembre 2013 •(462-468)

RePORTE DE CASO

\title{
EMBARAZO GEMELAR MONOCORIÓNICO MONOAMNIÓTICO. PRESENTACIÓN DE TRES CASOS Y REVISIÓN DE LA LITERATURA
}

\section{Monochorionic monoamniotic twin pregnancy. Three cases presented and review of the literature}

\author{
Juliana Rodríguez-Castillo, $\mathrm{MD}^{1}$; Julián Delgado-Gutiérrez, $\mathrm{MD}, \mathrm{PhD}^{2}$; \\ Juan Carlos Quintero-Mejía, $M D^{3}$; Tatiana Peña-Ramírez, $M D^{3}$; \\ Sandra García-Novillo, $M^{4}$
}

Recibido: mayo 10/13 - Aceptado: noviembre 18/13

\section{RESUMEN}

Objetivo: realizar el reporte de casos de embarazo gemelar monocoriónico monoamniótico con entrecruzamiento de cordones y hacer una revisión de la literatura respecto al pronóstico de este tipo de gestación, seguimiento prenatal e intervenciones de manejo hasta su finalización.

Materiales y métodos: se presenta el reporte de tres casos que ocurrieron en el Hospital Universitario del Valle, Cali, Colombia, centro de referencia de nivel III del suroccidente colombiano, el cual atiende población embarazada de alto riesgo obstétrico. Se desarrolló una búsqueda de la literatura en la base de datos Medline/Pubmed publicada en español e inglés durante el periodo comprendido entre enero de 1990 a mayo 2013. Se utilizaron los términos: "gemelos", "gemelos monocigóticos", "embarazo múltiple", "embarazo gemelar". Se incluyeron artículos de revisión, reporte de casos y estudios de corte transversal.

1 Residente de segundo año de Ginecología y Obstetricia, Universidad del Valle. Cali, Colombia. julianalrc@hotmail.com

2 Profesor asistente, Departamento de Ginecología y Obstetricia, Universidad del Valle. Cali, Colombia. PhD Obstetricia y Ginecología, Universidad Autónoma de Barcelona, España.

3 Profesor hora cátedra, Departamento de Ginecología y Obstetricia, Universidad del Valle. Cali, Colombia.

3 Especialista en Ginecología y Obstetricia, Universidad del Valle. Cali, Colombia.
Resultados: se revisaron 23 artículos de los cuales 5 correspondieron a artículos de revisión, 7 reportes de casos y 11 estudios de corte trasversal. La información acerca de las tasas de mortalidad, evaluación prenatal, seguimiento, manejo de las complicaciones, momento y modo del nacimiento en gemelos monoamnióticos es controversial.

Conclusión: la gestación gemelar monocorial monoamniótica constituye un evento poco frecuente. Esta condición está asociada con múltiples complicaciones obstétricas que incluyen la muerte fetal relacionada con accidentes de los cordones umbilicales (entrecruzamientos). El objetivo del cuidado prenatal constituye la prevención de la mortalidad fetal, por lo que se sugiere la realización continua de pruebas de bienestar fetal. Se ha propuesto la terminación del embarazo en la semana 34 de gestación por operación cesárea, previa administración de esteroides.

Palabras clave: gemelos, gemelos monocigóticos, embarazo múltiple, embarazo gemelar.

\section{ABSTRACT}

Objective: To report cases of monochorionic monoamniotic twin pregnancy with cord entanglement, and conduct a review of the literature regarding the prognosis of this type of gestation, 
prenatal follow-up and management interventions through to delivery.

Materials and methods: We report 3 cases that presented to Hospital Universitario del Valle, Cali, Colombia, a level III referral center in Southwestern Colombia that provides healthcare services to pregnant women with a high obstetric risk. We conducted a search in the literature in the Medline/ Pubmed database published in Spanish and English between January 1990 and May 2013. The terms used were "twins", "monozygotic twins", "multiple pregnancy", “twin pregnancy”. Review articles, case reports and cross-sectional studies were included. Results: Overall, 23 articles were reviewed, including 5 review articles, 7 case reports and 11 cross-sectional studies. Information on mortality rates, pre-natal assessment, follow-up, management of complications, time and mode of birth in monoamniotic twins is controversial.

Conclusion: Monochorial monoamniotic twin pregnancy is an infrequent event. This condition is associated with multiple obstetric complications, including fetal demise associated with umbilical cord accidents (entanglement). The goal of prenatal care is to prevent fetal death, hence the suggestion of continuous testing for fetal wellbeing. Termination of pregnancy has been proposed at week 34 of gestation through cesarean section after steroid administration.

Key words: Twins, monozygotic twins, multiple pregnancy, twin pregnancy.

\section{INTRODUCCIÓN}

Los embarazos gemelares corresponden a 1 de cada 80 embarazos, ellos contribuyen con una mortalidad perinatal 3 veces mayor que en embarazos simples y una elevada morbilidad con 10 veces más riesgo de lesiones neurológicas (1). Una tercera parte de los embarazos gemelares son monocigóticos y de estos solo un 1 a 2\% son monocoriónicos monoamnióticos (cerca de 1 de cada 10.000 embarazos), resultante de la división tardía del desarrollo del embrión entre el día 8 y el día 12 posfertilización (2).
El diagnóstico se realiza por ecografía de rutina de 11-14 semanas de gestación por los hallazgos de dos polos fetales, una placenta única no fusionada y la ausencia de membrana amniótica divisoria (3). En la ecografía de segundo o de tercer trimestre se pueden evidenciar los entrecruzamientos de los cordones y la ausencia de la membrana amniótica en fetos del mismo sexo. El diagnóstico final debe ser realizado por la valoración patológica de la placenta.

Se ha informado una tasa de mortalidad perinatal alta (20-50\%) atribuida a prematuridad, malformaciones congénitas, entrecruzamientos de los cordones umbilicales y síndrome de transfusión feto-fetal (4). La mortalidad perinatal aumenta con el embarazo siendo progresivamente mayor en el tercer trimestre (5).

Teniendo en cuenta las múltiples controversias existentes respecto al seguimiento prenatal, intervenciones de manejo y tiempo de finalización de este tipo de gestación múltiple, y siendo sus complicaciones de gran impacto en la morbimortalidad perinatal, se presenta este reporte de casos con el objetivo de hacer una revisión de la literatura respecto al pronóstico de este tipo de gestación, seguimiento prenatal e intervenciones de manejo hasta su finalización. Se propone un algoritmo de evaluación y seguimiento como herramienta para la toma de decisiones por parte del especialista en ginecología y obstetricia que se vea enfrentado a esta condición.

\section{REPORTE DE CASOS}

Caso 1. Paciente de 14 años, primigestante, quien fue remitida por embarazo gemelar monocoriónico monoamniótico de 31 semanas. Se realizó ecografía observando entrecruzamiento de cordones. Se decidió hospitalizar para monitorización estrecha con pruebas de bienestar fetal. Cuatro días después, la paciente inició actividad uterina y progresión de los cambios cervicales por lo que se decidió programar para cesárea. El primer neonato, con un peso de 1.750 g requirió manejo durante una semana en la unidad de cuidados intensivos neonatal por 
enfermedad de membrana hialina con evolución adecuada. El segundo gemelo pesó 1.825 g y tuvo evolución satisfactoria. Ambos neonatos se encuentran con la madre.

Caso 2. Paciente de 18 años G1P0, quien fue remitida a las 30 semanas para toma de ecografía. En ultrasonido realizado a las 21 semanas se reportó un embarazo gemelar monocorial monoamniótico, con crecimiento de ambos fetos en percentiles adecuados. En la nueva ecografía en la institución se corroboró el diagnóstico, además de la presencia de entrecruzamiento de los cordones. Se hospitalizó a la semana 31 para monitorización con pruebas de bienestar fetal y evaluación ecográfica cada 2-3 días. A las 34 semanas uno de los fetos presentó bradicardia fetal por lo que se decidió programar para cesárea. El primer gemelo pesó $1.750 \mathrm{~g}$, el segundo recién nacido pesó $1.570 \mathrm{~g}$ y requirió manejo intensivo por dos días por enfermedad de membrana hialina. Ambos se encuentran con la madre.

Caso 3. Paciente de 29 años G3P1C1 remitida del control prenatal por embarazo gemelar monocoriónico monoamniótico de 26 semanas y evidencia de cordones entrecruzados. Se ordenó el siguiente plan de manejo: maduración pulmonar, hospitalización preventiva con alto riesgo obstétrico a la semana 28 para vigilancia, y seguimiento estricto de crecimiento fetal, pruebas de bienestar diarias, ecografía obstétrica semanal y finalización por vía alta a las 32 semanas. A la semana 29 se presentó bradicardia fetal severa del primer gemelo. Se hizo diagnóstico de estado fetal no satisfactorio, por lo que se decidió programar para cesárea. El primer recién nacido pesó 1.142 g y el segundo 1.260 g. Los neonatos requirieron manejo en la unidad de cuidado intensivo neonatal durante 40 días. Ambos neonatos están en casa con la madre (figuras 1a, b; 2a, b, c).

La vigilancia fetal después de la semana 28 de embarazo en estas pacientes consistió en monitorización fetal anteparto dos veces al día y perfil biofísico fetal diario, lo que permitió detectar bradicardia fetal en dos de los casos y decidir la finalización inmediata del embarazo para evitar la muerte intrauterina. Se requirió manejo complementario con la unidad de cuidados intensivos neonatales.

\section{MATERIALES Y MÉTODOS}

La revisión de la literatura se desarrolló mediante una búsqueda en la base de datos Medline/Pubmed publicada en español e inglés durante el periodo
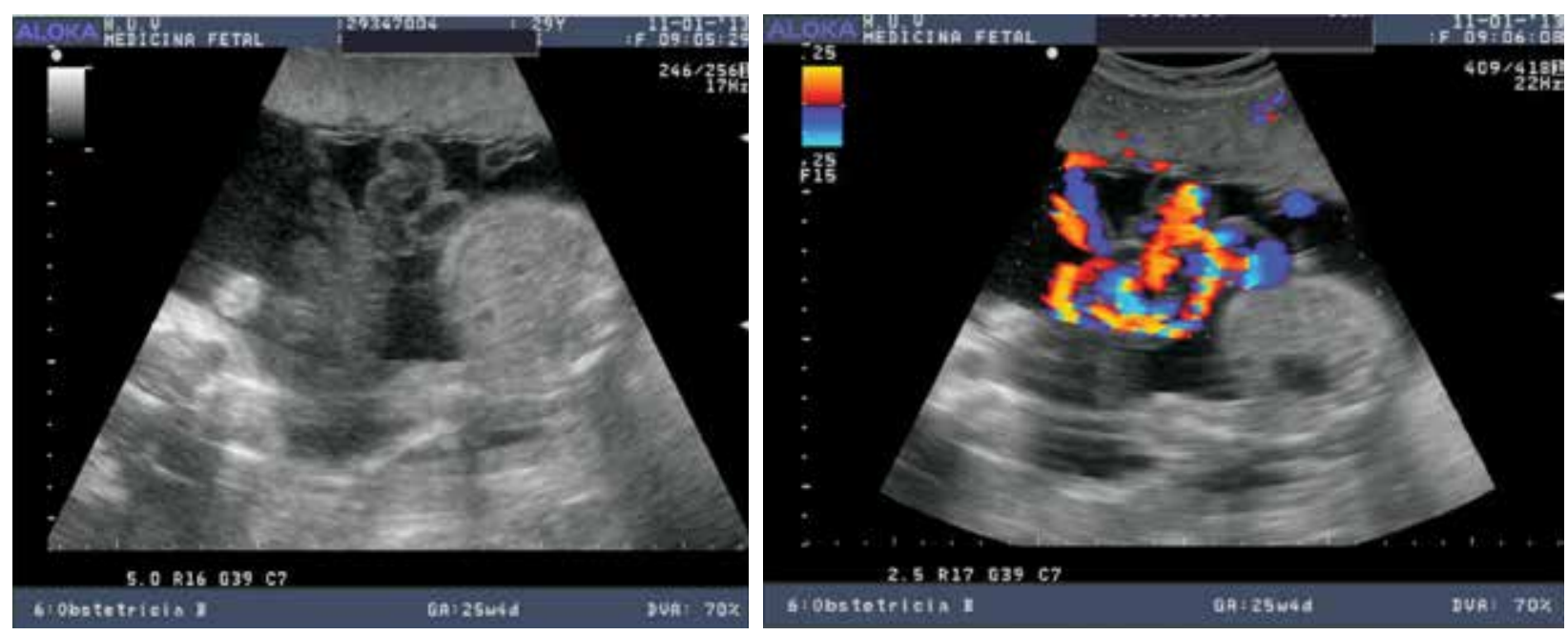

Figura 1a y b. Ecografía en la que se observa el entrecruzamiento de los cordones umbilicales (caso 3)

1a. Se observa el acúmulo de vasos umbilicales en la cavidad amniótica única 1b. Entrecruzamientos de cordones umbilicales resaltados con la exploración Doppler color 
Figuras 2a, b, c. Imágenes de la placenta y los cordones umbilicales del caso 3.

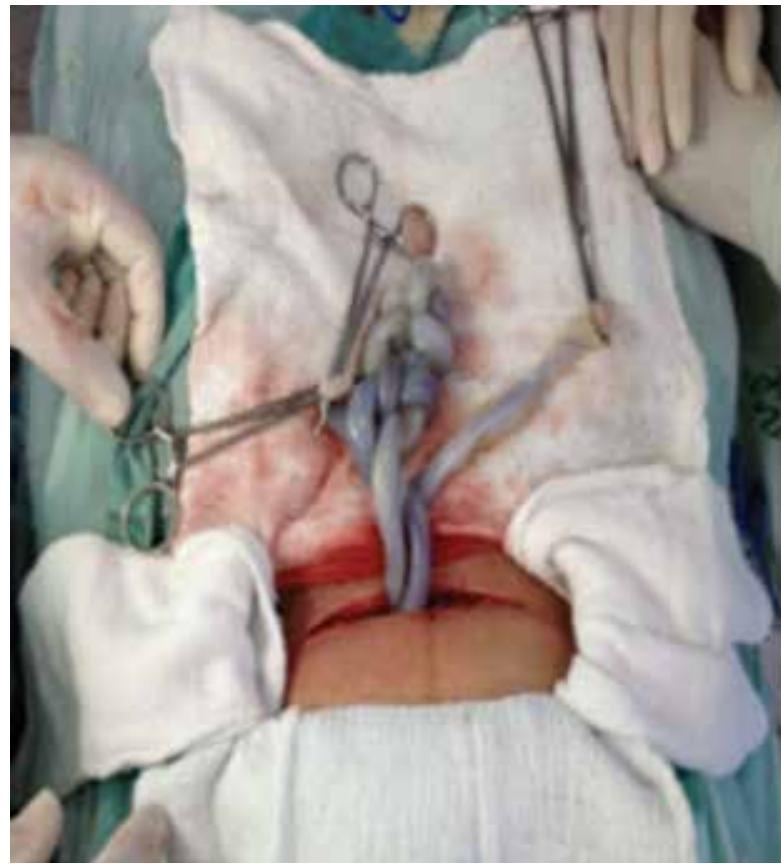

2a. Operación cesárea. Imagen de los entrecruzamientos de los cordones umbilicales previo a la extracción de la placenta

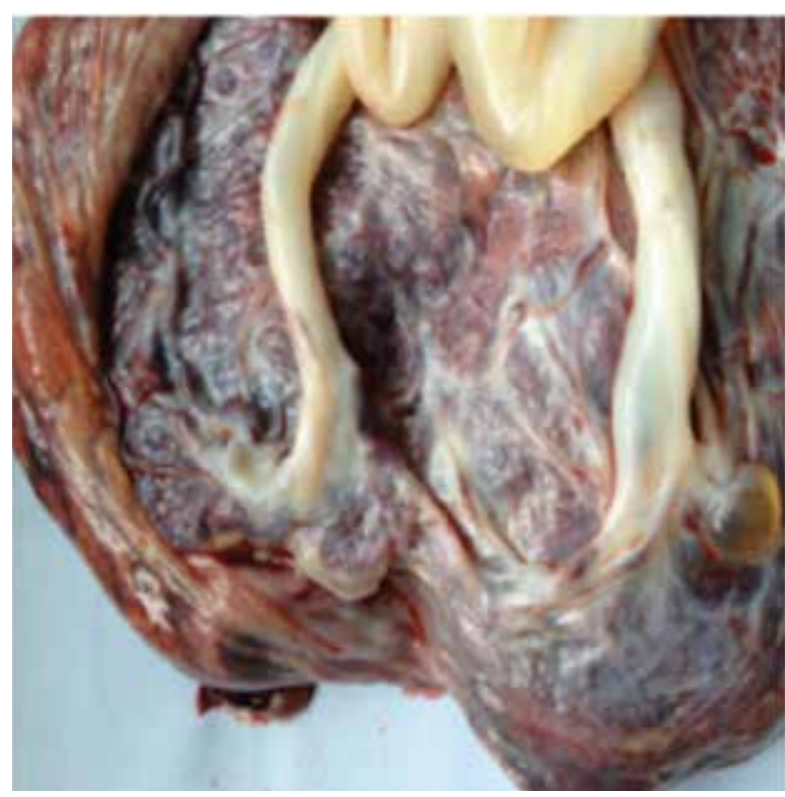

2c. Aproximación en el sitio de las inserciones de los cordones umbilicales. Se observa la distribución placentaria y la ausencia de ecuador amniótico

comprendido entre enero de 1990 a mayo de 2013. Se utilizaron los términos: "gemelos", "gemelos monocigóticos”, “embarazo múltiple”, “embarazo

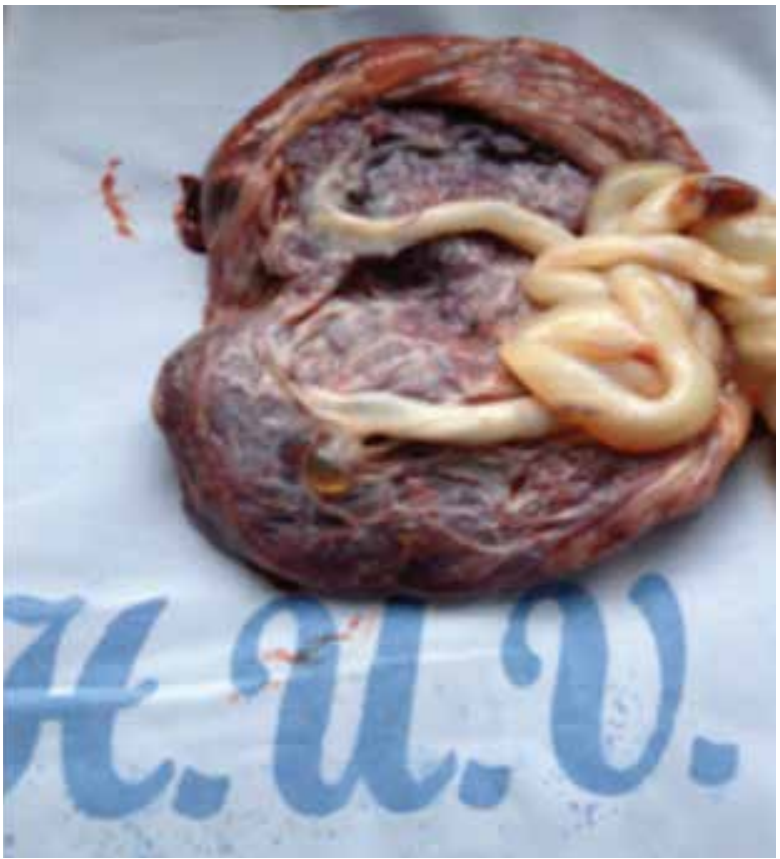

2b. Placenta, embarazo monocorial monoamniótico, con los entrecruzamientos de los cordones umbilicales

gemelar”. Se incluyeron artículos de revisión, reporte de casos y estudios de corte transversal que mencionaran el embarazo gemelar monocoriónico monoamniótico, la frecuencia de sus complicaciones, el seguimiento prenatal y las intervenciones de manejo.

Aspectos éticos. Se solicitó autorización de las participantes respecto a la divulgación de los datos de la historia clínica, extracción de las imágenes de la base de datos de ecografía y la toma de fotografías mediante consentimiento informado. Se garantizó confidencialidad en la información, protegiendo los derechos de las pacientes durante todo el proceso.

\section{RESULTADOS}

Como resultado de la revisión de la literatura se seleccionaron 23 artículos de interés, 5 correspondieron a artículos de revisión de la literatura, 7 reportes de casos y 11 experimentos clínicos de los cuales 10 se relacionaron con la vigilancia fetal anteparto, 7 con las complicaciones y 6 con el entrecruzamiento de cordones. 


\section{DISCUSIÓN}

La información acerca de la evaluación prenatal y el momento y modo del nacimiento en gemelos monoamnióticos, así como de las tasas de mortalidad es controversial y consiste en pocos estudios prospectivos, pequeños informes retrospectivos y algunas series de casos.

Pronóstico. Se conoce que los embarazos gemelares monocoriales monoamnióticos tienen una alta tasa de complicaciones tanto maternas como perinatales (6-8): en la literatura se ha reportado una incidencia de trabajo de parto pretérmino que varía del 50 hasta $80 \%$, como el que ocurrió en uno de los casos presentados (9).

El entrecruzamiento de los cordones es otra de las complicaciones reportadas y ocurre en alrededor del 42 al 80\% de los casos. Se pueden producir lesiones neurológicas cuando hay una compresión intermitente del cordón, y cuando esta se vuelve constante produce la muerte (10). En los tres casos presentados se evidenció que los cordones se encontraban entrecruzados, tanto por ecografía como al momento del nacimiento.

El diagnóstico de entrecruzamiento de los cordones antenatalmente en una revisión de 133 embarazos monoamnióticos se relacionó con menor edad gestacional al momento del parto $(30,4$ frente a 32,6$)$, pero se encontró también que estos pacientes tenían menor estancia hospitalaria en la unidad de cuidados intensivos neonatal (11). Una reciente revisión sistemática realizada por Rossi et al., comparó en 4 estudios el resultado perinatal de los embarazos complicados con entrecruzamiento de cordón frente a los controles. Se observó que no hubo una diferencia significativa respecto a tasa de sobrevida global y morbilidad neonatal (12).

Una herramienta útil para el diagnóstico y seguimiento es el Doppler fetoplacentario. El mapeo con flujo color está asociado con un valor predictivo de $89 \%$ para el diagnóstico de entrecruzamiento de cordones (13). Respecto al seguimiento, se realiza Doppler fetal con evaluación de la onda de flujo de la arteria umbilical: un aumento en el índice de pulsatilidad o un flujo diastólico ausente o reverso en diástole pueden sugerir compresión del cordón $(14,15)$.

El síndrome de transfusión feto-fetal es otra complicación que se describe en un 15\% de todos los embarazos monocoriónicos, pero con menor incidencia en los monoamnióticos debido a la presencia de anastomosis arterio-arteriales en la circulación placentaria que compensan las anastomosis veno-arteriales $(16,17)$.

Las gestaciones gemelares monoamnióticas también se pueden complicar con la presencia de anomalías congénitas, discordancia fetal, ruptura prematura de membranas y prolapso de cordón en el parto vaginal.

Seguimiento. Existe discusión en cuanto a la frecuencia de la monitorización y el tiempo de finalización del embarazo. La decisión debe ser tomada con base en la morbilidad y la mortalidad por la edad gestacional, y el riesgo de continuar el embarazo según la experiencia de cada institución (18). Se estima que el 25\% de todos los embarazos monoamnióticos tendrían al menos una pérdida fetal después de las 20 semanas de gestación.

Un estudio encontró que las pacientes hospitalizadas para monitorización continua tuvieron mayor incidencia de cesárea por estados fetales no tranquilizadores frente a las pacientes manejadas de manera ambulatoria, pero con una gran disminución de la mortalidad fetal entre las pacientes hospitalizadas (0 frente a 14,8\%), lo mismo que en la morbilidad fetal compuesta (29 frente a 50\%) (19).

La evidencia disponible hasta la fecha no permite definir cuál debe ser el momento del parto. Algunos autores sugieren que debido al mayor riesgo de muerte fetal intrauterina con respecto a otros tipos de gemelos, y a eventos agudos relacionados con el cordón umbilical secundario a entrecruzamientos, se debe considerar la finalización de los embarazos gemelares monocoriales no-complicados lo más temprano posible, a fin de limitar los eventos causados en el gemelo superviviente si uno fallece in utero en el tercer trimestre (20). Estos protocolos 
han sido sugeridos en numerosas estrategias para reducir la alta mortalidad perinatal, como por ejemplo, el nacimiento pretérmino electivo, el cual se debe realizar entre los límites de 32 a 35 semanas, por operación cesárea, previa maduración pulmonar fetal con corticoides (21). Sin embargo, se deberá tomar en cuenta la morbimortalidad neonatal que tenga cada institución (22). En la mayoría de informes hay una preferencia por el parto por cesárea para evitar el riesgo de accidentes de cordón en el parto y la mal presentación del segundo gemelo. El principal problema es que el cordón del segundo gemelo prolapsa al lado del primero (23).

Demaria et al. proponen que estos casos deben ser admitidos al hospital después de haber establecido la viabilidad para realizar cardiotocografía varias veces por día. Esto podría prevenir algunas muertes fetales, pero no suprime las muertes intrauterinas derivadas de los accidentes de cordón que pueden ocurrir sin previo aviso. Igualmente, la monitorización intensiva por 6 a 10 semanas en el hospital tiene un costo económico y social considerable y parece aumentar la incidencia de nacimiento urgente por estado fetal no satisfactorio (23).

En los casos reportados de nuestra institución se realizó maduración pulmonar y hospitalización preventiva para vigilancia fetal, y aunque esta fue menos estrecha respecto a la recomendaciones encontradas en la literatura (monitorización fetal anteparto dos veces al día y perfil biofísico diario después de la semana 28), en dos de los casos se pudo hacer el diagnóstico de estado fetal no satisfactorio, llevando las pacientes a cesárea de forma oportuna y no se presentó ningún caso de mortalidad fetal o neonatal.

\section{CONCLUSIONES}

De acuerdo con la revisión de la literatura y los resultados en los reportes de casos presentados, se podría sugerir que la estrategia de maduración pulmonar y hospitalización preventiva con vigilancia fetal estrecha tiene un impacto importante en la reducción de la mortalidad fetal, aunque representa mayores costos para el servicio de salud respecto al manejo ambulatorio. El tiempo del parto es un balance entre el riesgo de prematuridad con sus secuelas cerebrales y el de morbilidad y mortalidad fetal a diferentes edades gestacionales. La decisión de finalización electiva pretérmino debe estar basada en la estadística de la unidad neonatal de cada institución.

Se propone un algoritmo de manejo para las gestaciones monocoriónicas monoamnióticas con entrecruzamiento de cordones basado en la revisión realizada (figura 3).

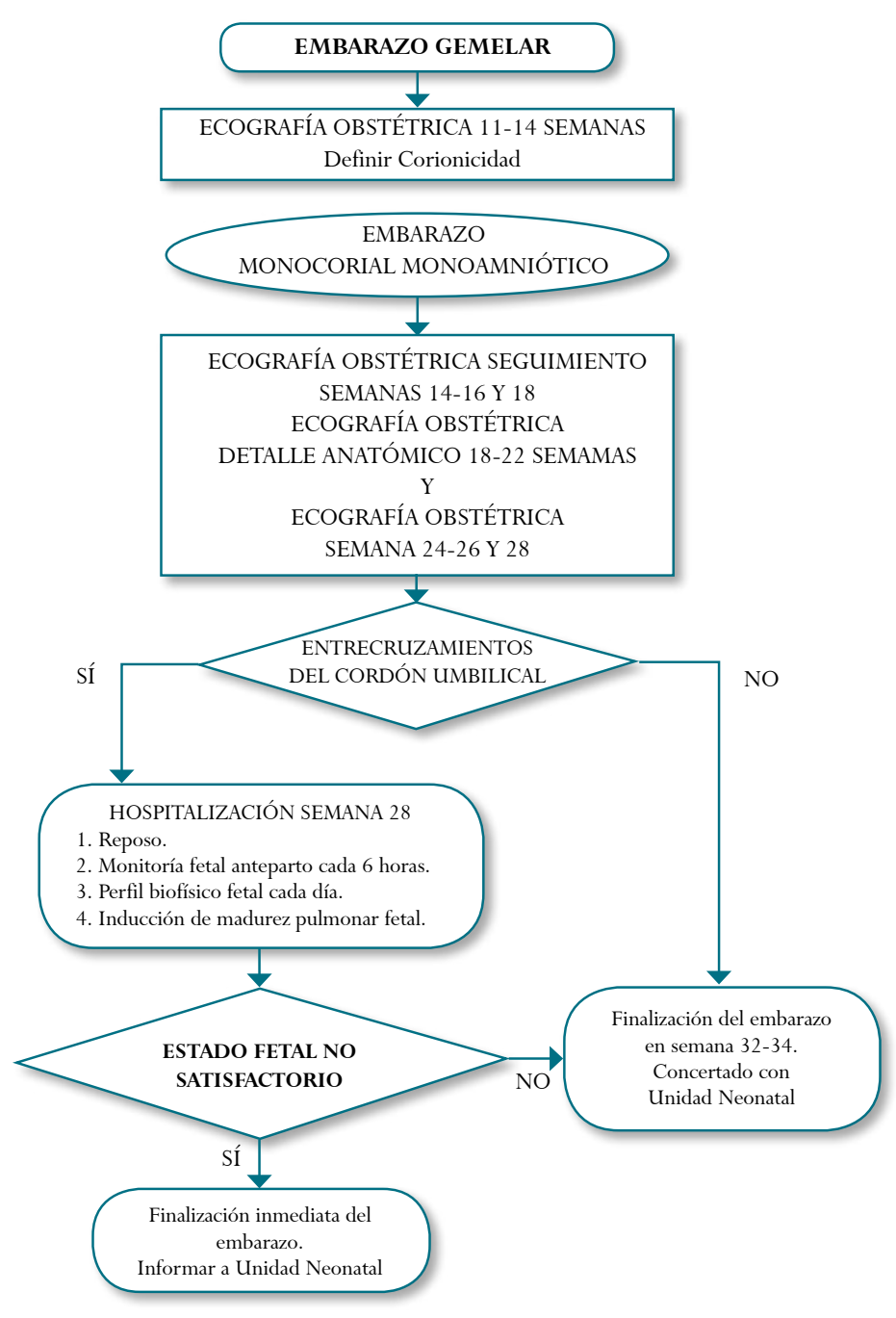

Figura 3. Propuesta de algoritmo de manejo de embarazo gemelar monocoriónico monoamniótico con entrecruzamiento de cordones 


\section{REFERENCIAS}

1. Hall JG. Twinning. Lancet. 2003;362:735-43.

2. Dickinson J. Monoamniotic twin pregnancy: a review of contemporary practice. Aust NZ J Obstet Gynaecol. 2005;45:474-8.

3. Sebire NJ, Souka A, Skentou H, Geerts L, Nicolaides KH. First-trimester diagnosis of monoamniotic twin pregnancies. Ultrasound Obstet Gynecol. 2000;16:223-5.

4. Roque H, Gillen-Goldstein J, Funai E, Young BK, Lockwood CJ. Perinatal outcomes in monoamniotic gestations. J Matern Fetal Neonatal Med. 2003;13: 414-21.

5. Carr SR, Aronson MP, Coustan DR. Survival rates of monoamniotic twins do not decrease after 30 weeks' gestation. Am J Obstet Gynecol. 1990;163:719-22.

6. Allen VM, Windrim R, Barrett J, Ohlsson A. Management of monoamniotic twin pregnancies: a case series and systematic review of the literature. BJOG. 2001;108:931-6.

7. Beasley E, Megerian G, Gerson A, Roberts NS. Monoamniotic twins: case series and proposal for antenatal management. Obstet Gynecol. 1999;93:130-4.

8. Cordero L, Franco A, Joy SD. Monochorionic monoamniotic twins: neonatal outcome. J Perinatol. 2006;26:170-5

9. Suzuki S, Kaneko K, Shin S, Araki T. Incidence of intrauterine complications in monoamniotic twin gestation. Arch Gynecol Obstet. 2001;265:57-9.

10. Overton TG, Denbow ML, Duncan KR, Fisk NM. First trimester cord entanglement in monoamniotic twins. Ultrasound Obstet Gynecol. 1999;13:140-2.

11. Arabin B, Laurini RN \& van Eyck J. Early prenatal diagnosis of cord entanglement in monoamniotic multiple pregnancies. Ultrasound Obstet Gynecol. 1999;13:181-6.

12. Rossi AC, Prefumo F. Impact of cord entanglement on perinatal outcome of monoamniotic twins: a systematic review of the literature. Ultrasound Obstet Gynecol. 2013;41:131-5.
13. Ertan AK, Schmidt W. [Umbilical cord entanglement and color-coded Doppler ultrasound]. Geburtshilfe Frauenheildk 1994;54:196-203.

14. Abuhamad AZ, Mari G, Copel JA, et al. Umbilical artery flow velocity waveforms in monoamniotic twins with cord entanglement. Obstet Gynecol. 1995;86:674-77.

15. Belfort MA, Moise KJ, Kirshon B, Saade G. The use of color flow Doppler ultrasonography to diagnose umbilical cord entanglement in monoamniotic twin gestations. Am J Obstet Gynecol, 1993;168:601-4.

16. Umur A, van Gemert MJC, Nikkels PGJ. Monoamniotic-versus diamniotic-monochorionic placentas: Anastomoses and twin-twin transfusion syndrome. Am J Obstet Gynecol. 2003;189:1325-9.

17. Pasquini L, Wimalasundera RC, Fisk NM. Management of other complications specific to monochorionic twin pregnancies. Best Pract Res Clin Obstet Gynaecol. 2004;18:577-99.

18. Heyborne KD, Porreco RP, Garite TJ, Phair K, Abril D. Improved perinatal survival of monoamniotic twins with intensive inpatient monitoring. Am J Obstet Gynecol. 2005;192:96-101.

19. Ezra Y, Shveiky D, Ophir E, Nadjari M, Eisenberg VH, Samueloff A, Rojansky N. Intensive management and early delivery reduce antenatal mortality in monoamniotic twin pregnancies. Acta Obstet Gynecol Scand. 2005;84:432-5

20. Su LL. Monoamniotic twins: diagnosis and management. Acta Obstet Gynecol Scand. 2002;81:995-1000.

21. Tessen JA, Zlatnik FJ. Monoamniotic twins: a retrospective controlled study. Obstet Gynecol. 1991;77:832-4.

22. Rodis JF, McIlveen PF, Egan JF, Borgida AF, Turner GW, Campbell WA. Monoamniotic twins: improved perinatal survival with accurate prenatal diagnosis and antenatal fetal surveillance. Am J Obstet Gynecol. 1997;177:1046-9.

23. Demaria F, Goffinet F, Kayem G, Tsatsaris V, Hessabi M, Cabrol D. Monoamniotic twin pregnancies: antenatal management and perinatal results of 19 consecutive cases. BJOG. 2004;111:22-6. 